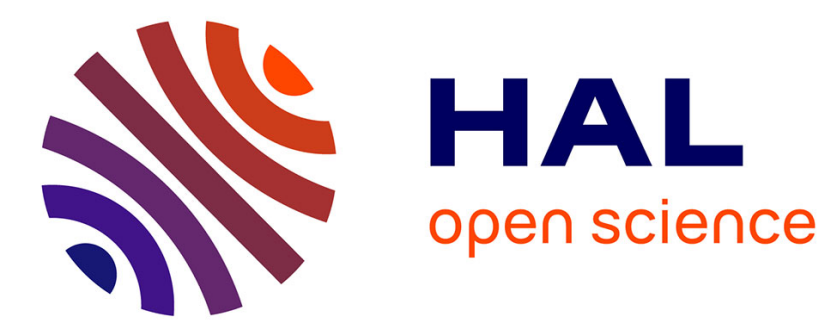

\title{
Diagramme des phases et domaine de formation de verres dans le système pseudo-binaire GeSe2-Te
}

S. Bordas, M. Geli, J. Casas-Vázquez, N. Clavaguera, M. T. Clavaguera-Mora

\section{To cite this version:}

S. Bordas, M. Geli, J. Casas-Vázquez, N. Clavaguera, M. T. Clavaguera-Mora. Diagramme des phases et domaine de formation de verres dans le système pseudo-binaire GeSe2-Te. Revue de Physique Appliquée, 1977, 12 (5), pp.677-680. 10.1051/rphysap:01977001205067700 . jpa-00244224

\section{HAL Id: jpa-00244224 https://hal.science/jpa-00244224}

Submitted on 1 Jan 1977

HAL is a multi-disciplinary open access archive for the deposit and dissemination of scientific research documents, whether they are published or not. The documents may come from teaching and research institutions in France or abroad, or from public or private research centers.
L'archive ouverte pluridisciplinaire HAL, est destinée au dépôt et à la diffusion de documents scientifiques de niveau recherche, publiés ou non, émanant des établissements d'enseignement et de recherche français ou étrangers, des laboratoires publics ou privés. 


\title{
DIAGRAMME DES PHASES ET DOMAINE DE FORMATION DE VERRES DANS LE SYSTĖME PSEUDO-BINAIRE GeSe -Te $^{-T}$
}

\author{
S. BORDAS, M. GELI, J. CASAS-VÁZQUEZ
}

Departamento de Termología, Universidad Autónoma de Barcelona, Bellaterra, Espagne

\section{N. CLAVAGUERA}

Facultad de Física, Universidad de Barcelona, Espagne

\section{T. CLAVAGUERA-MORA}

Departamento de Física Fundamental, Universidad de Valladolid, Espagne

\begin{abstract}
Résumé. - Nous obtenons le diagramme de phases et le domaine de formation de verres par trempe à l'air dans ce système par ATD en utilisant comme méthodes complémentaires la microscopie électronique à balayage et la diffraction aux rayons $\mathrm{X}$. Nous nous intéressons aux liens qui peuvent s'établir entre, d'une part, les propriétés des verres observées par ATD, et, d'autre part, le diagramme de phases, non seulement en ce qui concerne le domaine de formation de verres, mais aussi à la séparation de phases liée à l'existence d'une démixion en phase liquide.
\end{abstract}

Abstract. - The phase diagram and the glass-forming region by quenching in air have been obtained for this system for DTA using scanning electron microscopy and X-ray diffraction as complementary methods. We attempted to relate the observed DTA behaviour of the glasses with the corresponding phase diagram obtained. In this sense particular attention has been directed not only to glass-forming regions but also to phase separation coming from liquid-immiscibility, and to the temperatures of the transformations.

1. Introduction. - L'étude des matériaux chalcogénures vitreux se poursuit depuis quelques années par suite de leur importance comme matériaux électroniques. Etant donné l'intérêt de connaître les conditions nécessaires pour l'obtention de ces matériaux en phase non cristalline, ceci a conduit à établir les domaines de vitrification pour plusieurs systèmes ternaires. De même, récemment, l'étude du diagramme de phases de quelques systèmes chalcogénures pseudo-binaires a permis de préciser la stabilité et l'évolution de ces verres d'une manière plus générale.

Des études précédentes sur le système Ge-Se-Te [1] nous ont permis de prévoir l'existence du système pseudo-binaire $\mathrm{GeSe}_{2}$-Te dont nous présentons ici le diagramme de phases. Nous présentons aussi le domaine de formation de verres par trempe à l'air, car nous constatons des différences entre le domaine de formation de verres dans le système Ge-Se-Te obtenu par A. V. Pazin et al. [2] et nos résultats. A part l'intérêt d'étudier le système pseudobinaire, notre but était au départ de trouver les liens qui existent entre le diagramme de phases et les propriétés des verres obtenus par trempe et aussi déceler les causes de variation des limites du domaine de formation de verres pour des conditions de préparation très proches.
2. Méthodes expérimentales. - Les échantillons de l'ordre d'un gramme : sont obtenus à partir des proportions appropriées des éléments (pureté $99,999 \%$ ) qui sont mis en ampoules de quartz (diamètre intérieur $5 \mathrm{~mm}$, épaisseur $1 \mathrm{~mm}$ ), scellées sous vide, puis portées à $1000^{\circ} \mathrm{C}$ et constamment agitées pendant $12 \mathrm{~h}$. La masse est ensuite trempée à l'air. L'analyse par diffraction X sur chambre Guinier de Wolff $\left(\mathrm{K}_{\alpha} \mathrm{du}\right.$ cuivre) permet de déterminer si l'échantillon est non cristallin. Les ATD et ATG sont effectuées simultanément à l'aide d'un appareil STA 429 Netzsch, sur des échantillons en poudre de $200 \pm 0,1 \mathrm{mg}$ et sous atmosphère dynamique d'argon. On utilise le carbure de silicium comme matériau de référence. La vitesse d'analyse est constante et égale à $5^{\circ} \mathrm{C} / \mathrm{min}$.

Un deuxième type d'échantillon est obtenu à partir de la poudre chauffée au-dessus de la température de liquidus, par refroidissement à $5^{\circ} \mathrm{C} / \mathrm{min}$. sous atmosphère d'argon. Ce deuxième type d'échantillons est utile pour l'obtention du diagramme de phases car sauf pour le $\mathrm{GeSe}_{2}$ la perte de masse est négligeable dans l'intervalle de températures utilisées $\left({ }^{1}\right)$.

(1) Plus exactement la perte de masse est nulle jusqu'à $440^{\circ} \mathrm{C}$ et elle reste inférieure à $1 \%$ jusqu'à $560^{\circ} \mathrm{C}$. 
L'analyse de l'homogénéité des matériaux obtenus par trempe est réalisé à l'aide d'un microscope électronique à balayage muni d'analyseur EDAX.

3. Diagramme de phases. - la Ligne $\mathrm{GeSe}_{2}-\mathrm{Te}$ correspond a priori à une des lignes invariantes du système ternaire Ge-Se-Te. Le diagramme de phases, présenté sur la figure 1, a été construit à partir des

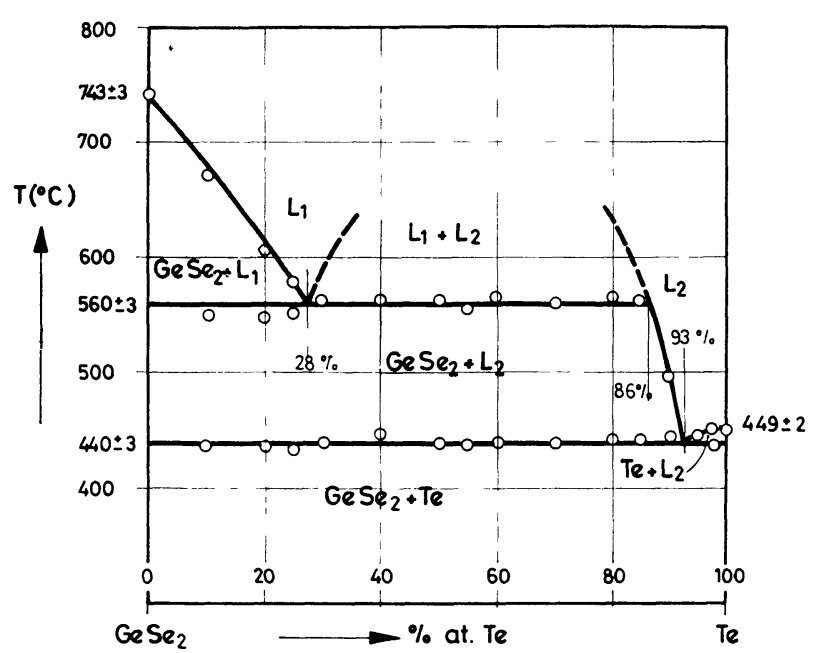

FIG. 1. - Diagramme de phases du système pseudo-binaire $\mathrm{GeSe}_{2}$-Te.

thermogrammes réalisés sur différentes compositions. Pour chaque composition étudiée on a effectué plusieurs cycles consécutifs d'ATD. L'analyse de cette figure nous permet de conclure $[3,4,5]$.

- Il existe une démixion en phase liquide pour des compositions comprises entre $28 \%$ at. et $86 \%$ at. de tellure $\left({ }^{2}\right)$, ce qui se manifeste par une réaction monotectique à $560 \pm 3^{\circ} \mathrm{C}$ du type

$$
\mathrm{L}_{1} \rightarrow \mathrm{GeSe}_{2}+\mathrm{L}_{2} \text {. }
$$

- L'existence de cette monotexie à température constante prouve par elle-même que ce système a uniquement deux composants, le $\mathrm{Te}$ et le $\mathrm{GeSe}_{2}$.

- On obtient aussi un palier invariant entre le $\mathrm{GeSe}_{2}$ et le Te à la température de $440 \pm 3^{\circ} \mathrm{C}$ qui correspond à l'eutectique de composition $93 \%$ at. de tellure $\left({ }^{3}\right)$.

- Le fait que les températures de solidus et liquidus de ce diagramme soient supérieures à celles des compositions situées de part et d'autre de cette ligne sur le système ternaire [6] atteste l'existence d'un eutectique. Comme, par ailleurs, cette ligne invariante s'appuie sur un composé défini à fusion congruente et

(2) La détermination des compositions des limites de lacune de miscibilité, eutectique, domaine de formation de verres, etc. ont été effectuées à $\pm 1 \%$ at. de Te près.

(3) La détermination de cette composition présente des difficultés étant donné qu'il s'agit d'un eutectique dégénéré et elle a été effectuée par la méthode de Tamman. un corps simple, on en conclut que la ligne $\mathrm{GeSe}_{2}-\mathrm{Te}$ est une ligne pseudo-binaire.

4. Domaine de formation de verres par trempe à l'air. - Les analyses par ATD montrent que toutes les compositions ayant une concentration en tellure inférieure à 93 at. \% présentent un pic de recristallisation pour une vitesse de chauffe de $5^{\circ} \mathrm{C} / \mathrm{min}$., mais la transition vitreuse est appréciable seulement pour des compositions ayant une concentration en tellure inférieure à $50 \%$ at. Les températures de ces transformations sont présentées sur la figure 2 .

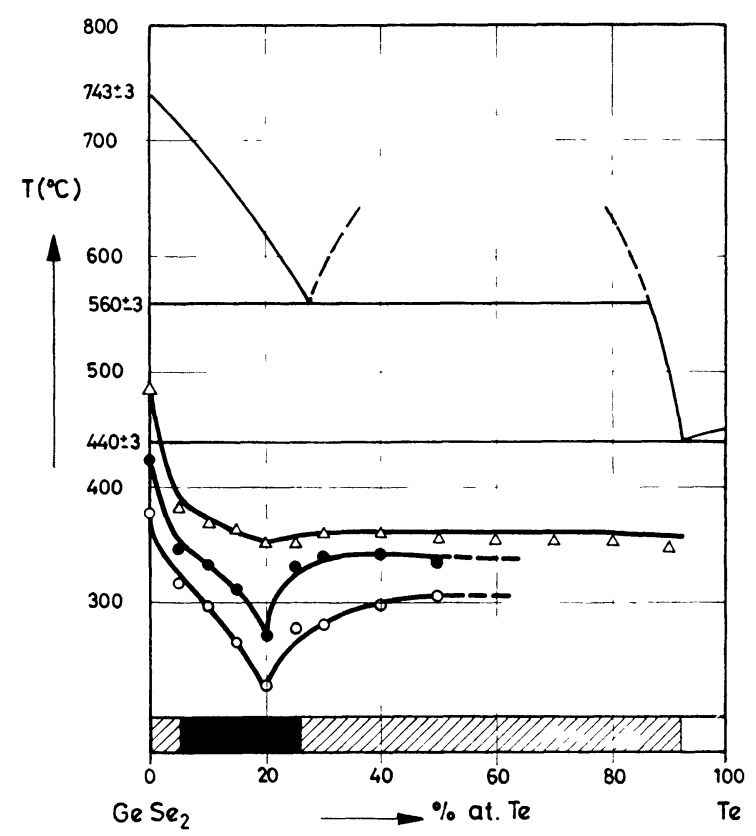

FIG. 2. - Températures des transformations propres à l'état vitreux : $\bigcirc$ : début de la transition vitreuse ; $\bullet$ : fin de la transition vitreuse ; $\triangle:$ début de recristallisation; et domaine de formation de verres. - région en noir : échantillon totalement non cristallin, - région en hachuré : échantillon partiellement cristallin.

Tous les échantillons de composition comprise entre $5 \%$ et $28 \%$ at. de tellure obtenus par trempe présentent un caractère totalement non cristallin. En effet, les diagrammes obtenus par diffraction $\mathrm{X}$ sur des échantillons de $\left(\mathrm{GeSe}_{2}\right)_{90} \mathrm{Te}_{10},\left(\mathrm{GeSe}_{2}\right)_{80} \mathrm{Te}_{20}$ et $\left(\mathrm{GeSe}_{2}\right)_{75} \mathrm{Te}_{25}$ présentent des halos très flous après des temps de pose de quelques heures, suffisants à l'obtention de diagrammes très nets lorsqu'il s'agit d'échantillons recristallisés. De même les thermogrammes respectifs montrent que pour ces compositions l'enthalpie de recristallisation à une valeur comparable à celle de fusion, ce qui n'est pas le cas pour des échantillons partiellement cristallins. On pourrait penser que ces résultats sont en désaccord avec les travaux de A. V. Pazin et al. mais il faut signaler que les méthodes de préparation ne sont pas identiques, la différence à notre avis la plus importante étant que ces auteurs préparent $5 \mathrm{~g}$ de substance tandis que nous en préparons $\sim 1 \mathrm{~g}$. 
Pour essayer de comprendre comment cette différence peut perturber les limites du domaine de formation de verres nous constatons d'abord qu'après refroidissement à $5{ }^{\circ} \mathrm{C} / \mathrm{min}$. toutes les compositions étudiées sont cristallines. Quelques expériences de refroidissement plus rapide nous ont permis de trouver que pour des compositions comprises entre $5 \%$ at. et $28 \%$ at. de tellure, la vitesse de refroidissement critique est située entre 1 et $10^{\circ} \mathrm{C} / \mathrm{s}$, tandis que cette vitesse est de 10 à $10^{2} \mathrm{o} / \mathrm{s}$ pour des compositions situées dans les intervalles $0-5 \%$ at. et $28-33 \%$ at. de tellure. Ces résultats permettent d'expliquer des variations du domaine de formation de verres suivant les conditions précises de trempe utilisées.

Ainsi donc, pour les conditions de trempe à l'air que nous avons utilisées, la région de formation de verres sur la ligne $\mathrm{GeSe}_{2}$-Te peut être décomposée en deux domaines suivant que les matériaux obtenus sont partiellement ou totalement non cristallins, comme il est indiqué sur la figure 2.

Nous constatons sur cette figure que les températures du début de la transition vitreuse et de la recristallisation ainsi que celles de la fin de la transition vitreuse restent à peu près constantes pour des compositions avec un contenu en tellure supérieur à $28 \%$ at. Nous avons donc séparation de phases provenant de la démixion en phase liquide. En effet, sur la figure 3

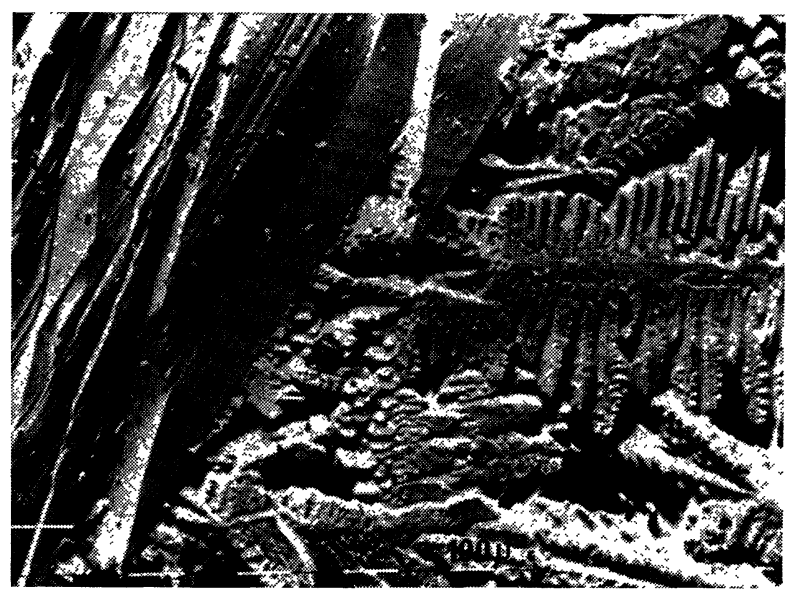

Fig. 3. - Microphotographie d'un échantillon de $\left(\mathrm{GeSe}_{2}\right)_{20} \mathrm{Te}_{80}$ avec séparation de phases vitreuse et cristalline.

nous présentons l'aspect au microscope électronique à balayage d'un échantillon de $\left(\mathrm{GeSe}_{2}\right)_{20} \mathrm{Te}_{80}$ où l'on observe clairement la séparation de phases prévue, avec une phase non cristalline (fracture vitreuse) et une phase cristalline de structure dendritique liée aux conditions de refroidissement. Sur les trois figures suivantes nous présentons l'analyse, sur cette surface, de la distribution de $\mathrm{Se}, \mathrm{Ge}$ et $\mathrm{Te}$, d'où l'on peut conclure que la phase non cristalline est de composition proche à $\mathrm{GeSe}_{2}$ tandis que la phase cristalline est proche au Te. L'application du modèle de Cahn et Charles [7] au système $\mathrm{GeSe}_{2}$-Te donne lieu à la figure 7

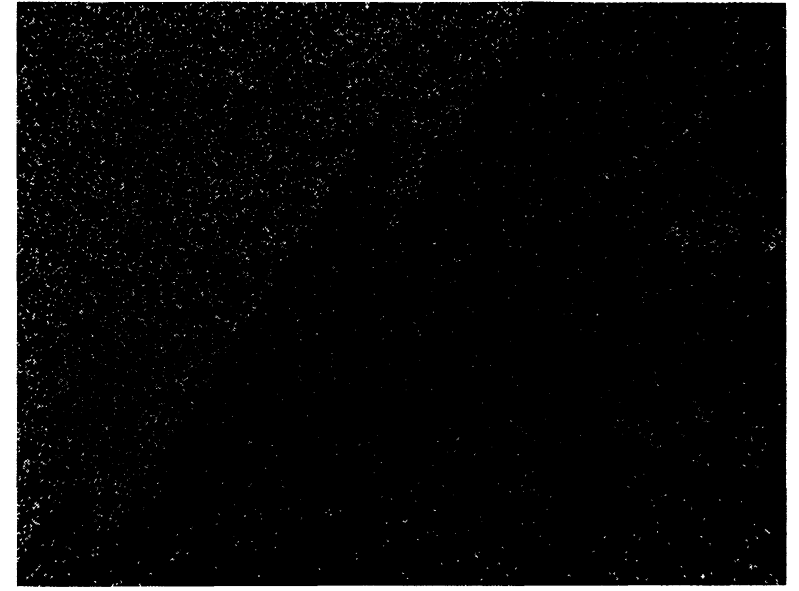

Fig. 4. - Analyse de la répartition de Ge.

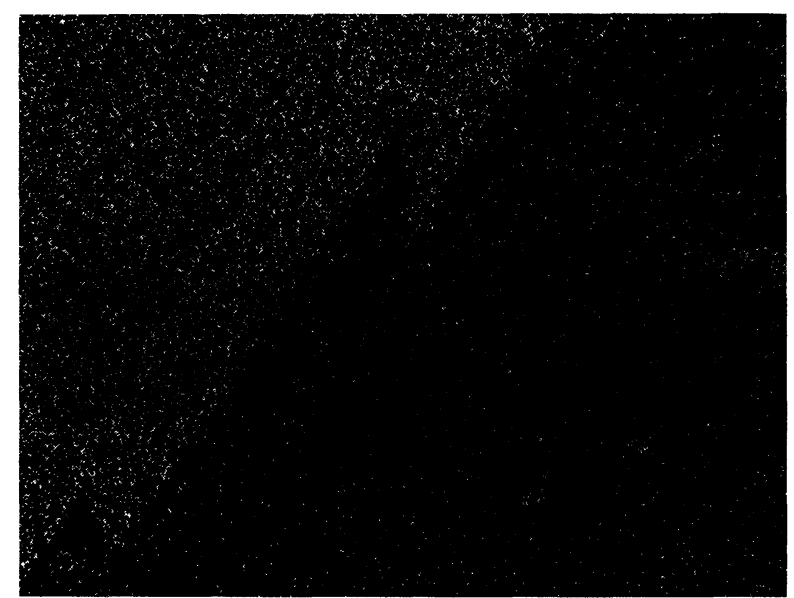

Fig. 5. - Analyse de la répartition de Se.

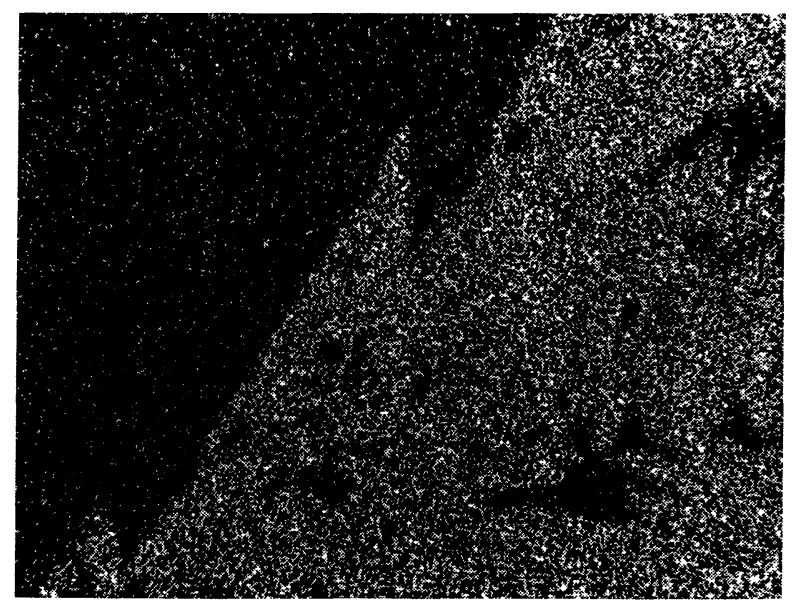

Fig. 6. - Analyse de la répartition de Te.

où nous avons représenté schématiquement pour les températures $T_{1}$ et $T_{2}$ de la figure $7 a$ la variation de l'enthalpie libre avec la composition (Fig. 7b). Après un refroidissement très rapide l'on peut obtenir à la température $T_{2}$ un équilibre métastable représenté par la ligne en pointillé de la figure $7 b$; mais si le 

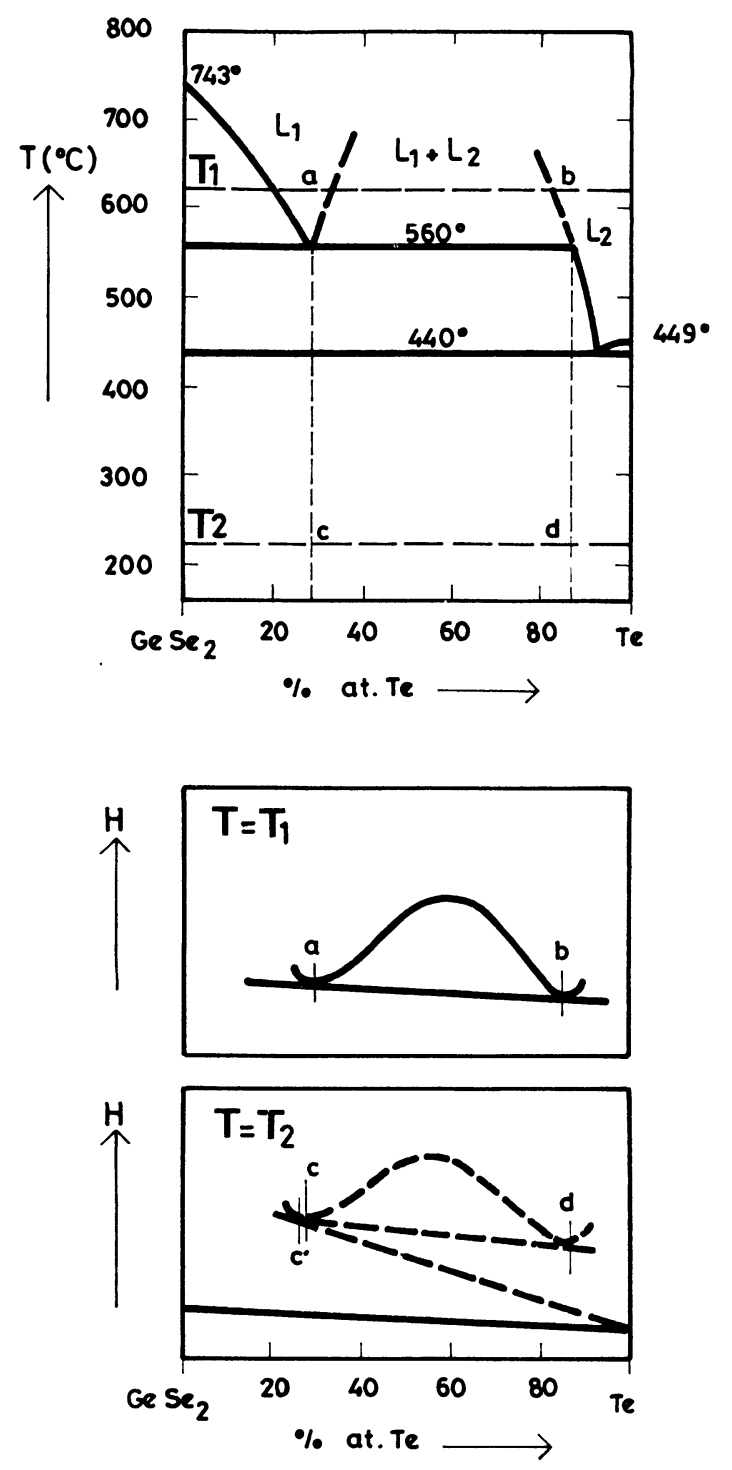

Fig. 7. - Diagramme de phases et énergies libres hypothétiques aux températures $T_{1}$ et $T_{2}$ dans le système $\mathrm{GeSe}_{2}$-Te.

refroidissement n'est pas suffisamment rapide pour empêcher la formation de $\mathrm{Te}$ cristallin le produit obtenu par trempe sera un mélange déphasé. On a porté aussi sur cette figure en tracé continu l'enthalpie libre correspondante à l'équilibre stable des deux phases cristallines $\mathrm{GeSe}_{2}$ et Te. Suivant ce modèle il est donc tout à fait raisonnable d'admettre que pour des compositions comprises entre $28 \%$ at. et $93 \%$ at. de tellure la trempe donne lieu à une phase non cristalline de composition proche à $28 \%$ at. de tellure (point c sur la figure 7) et du tellure cristallin. Cette interprétation explique la diminution de $\Delta C_{\mathrm{p}}$ à $T_{\mathrm{g}}$ et aussi de l'enthalpie de recristallisation quand on augmente la concentration en tellure, jusqu'à rendre cette variation difficile à apprécier.

5. Conclusions. - Dans cette étude nous avons cherché à mettre en évidence les liens qui existent entre le diagramme de phases et les propriétés des matériaux obtenus par trempe dans un système pseudo-binaire. Ces liens existent si la substance obtenue par refroidissement rapide garde un souvenir de son état antérieur. Les résultats obtenus appuient les conclusions suivantes :

- Il existe dans le système pseudo-binaire $\mathrm{GeSe}_{2}-\mathrm{Te}$ un large domaine de démixion en phase liquide.

- Cette démixion en phase liquide est responsable de la séparation de phases des matériaux obtenus par trempe et empêche que les substances de composition proche à l'eutectique soient des bons formateurs de verre.

- Le domaine de vitrification par trempe à l'air est très sensible à des faibles variations de la méthode de préparation. Il s'avère donc nécessaire de préciser la vitesse de refroidissement critique.

- L'ATD des matériaux obtenus par trempe est très utile car l'indépendence des températures de transition vitreuse et de recristallisation avec la composition, permet d'affirmer que l'échantillon se sépare en deux phases de composition constante.

Nous remercions les facilités accordées par le «Departamento de Cristalografía y Mineralogía» de la Faculté de Géologie de l'Université de Barcelone pour l'utilisation de la chambre Guinier de Wolff, et la «Cátedra de Metalúrgia de la E. T. S. I. I. » de l'Université Polytechnique de Barcelone pour l'utilisation du microscope à balayage.

\section{Bibliographie}

[1] Bordas, S., Clavaguera, N., Casas-Vázquez, J. and Clavaguera-Mora, M. T., Proc. 1st ESTA, Salford, England (1976) p. 426.

[2] Pazin, A. V., Obraztsov, A. A. and Borisova, Z. U., Izv. Akad. Nauk SSSR, Neor. Mater. 8 (1972) 247.

[3] Rollet, A. P. et Bouazir, R., L'Analyse Thermique (Gauthier-Villars, Paris) 1972, Tome I.
[4] Ferguson, F. D. and Jones, T. K., The phase rule (Butterworths and Co. Limited, London) 1966.

[5] Azoulay, R., Thibierge, H. and Brenac, A., J. Non Cryst. Solids 18 (1976) 33.

[6] Bordas, S., To be published.

[7] Cahn, J. W. and Charles, R. J., Phys. Chem. Glasses 4 (1965) 181. 